\title{
Editorial
}

\section{Message from Editor-in-chief}

Since the beginning of September last year, I have had the honor of serving as the editor-in-chief of MANUSYA: Journal of Humanities. With the release of this year's first issue, I would like to take this opportunity of sharing with all esteemed colleagues the important changes that our journal is going through.

First of all, I would like to express my deepest gratitude to our former editor-in-chief, Amara Prasithrathsint, for her dedication to the journal. Since 1996, MANUSYA under her leadership has been transformed into a truly international journal. Over the past few years, we have seen a steady rise in the number of submissions as well as an increase in author diversity. Annually, we are publishing over 20 articles by authors from various countries both inside and outside Southeast Asia. Furthermore, we are now indexed in various internationally recognized databases including ASEAN Citation Index (ACI), Modern Language Association (MLA) and Scopus. All this could not possibly have happened without her wisdom and hard work. On behalf of those of us who have professionally and intellectually benefited from MANUSYA, I would like to thank Amara for her 16 years of tremendous service to the humanities research community in Thailand, Southeast Asia and beyond.

The year 2021 is an exciting and challenging year for MANUSYA. With the growth of our journal, the editorial responsibilities have increased exponentially. To meet with the growing and increasingly diverse submissions, Kanya Wattanagun (Chulalongkorn University) and Treepon Kirdnak (Chulalongkorn University) have graciously agreed to join us as associate editors. The three of us will work closely together to ensure the high academic standard of MANUSYA.

Academically, we have revised our aim \& scope to reflect the gradual change in direction of our journal. This slightly shifted focus will be on Southeast Asian history, philosophy, religion, language, culture, literature, media and the arts. We still welcome submissions that are not strictly SEA-related, but priority will 
be given to research that has implications for Southeast Asia or approaches broader humanities issues from a Southeast Asian perspectives.

With the growing number of submissions comes another academic change. We are gradually increasing the annual number of published articles. This year we already have 27 articles lined up and we will reach our limit of 30 articles per volume next year. Unfortunately, this means fewer pages for each article. A new set of submission guidelines for authors is available on our website at http://www.manusya.journals.chula.ac.th/.

On the logistics side, we have partnered with Brill to achieve maximum distribution since 2019. While Chulalongkorn University retains responsibility and control over all the intellectual content of the journal, articles published in MANUSYA will be available free of charge through Brill's open access. In response to new trends in academic publishing, we are also working together to implement Advance Article publication, which ensures that articles accepted for the journal are available online within about 6-8 weeks of acceptance. We hope to have this new service in place by the beginning of 2022 .

Another big change is the switch from the Open Journal System to Editorial Manager, made available to us by the technical and financial support of the Thailand Citation Index (тсг) funded by Thailand Science Research and Innovation (TSRI). Starting from January 2021, all new submissions must be made through our submission portal at https:/www.editorialmanager.com/ manusya/. With this new journal management system, we hope to speed up the review process and further ensure transparency.

Lastly but not least, I would like to thank all those who have contributed to the success of MANUSYA. I would like to express my appreciation to past and present members of the Editorial Board for all their contributions. Moreover, my deepest gratitude goes to all the reviewers and guest editors whose expertise and generosity are at the very core of our success. I would like to thank Chulalongkorn University's Office of Research Affairs for its continuing financial support, which has kept MANUSYA free for both authors and readers. I would also like to thank our editorial assistants Chantharas Kanchanakool and Pimpat Chumkaew for their care in making MANUSYA an ever better journal and their tireless efforts in getting everything going.

Sincerely,

Pittayawat Pittayaporn

Editor-in-chief 